\title{
Estrategia metodológica basado en proyectos incide en el aprendizaje de matemáticas - nivel secundaria
}

\author{
Ana Maricela Jácome Vera \\ p7002291741@ucvvirtual.edu.pe \\ https://orcid.org/0000-0001-5118-4557 \\ Universidad César Vallejo, Piura - Perú \\ Lucía Espinoza Cedillo \\ espinozacedillo@hotmail.com \\ https://orcid.org/0000-0001-7217-215X \\ Universidad César Vallejo, Piura - Perú \\ César Balladares Atoche \\ cesarballadares@hotmail.com \\ https://orcid.org/0000-0001-8242-7742 \\ Universidad César Vallejo, Piura - Perú \\ Nora Mercedes Torres Torres \\ n.torrest2309@gmail.com \\ https://orcid.org/0000-0002-0623-1294 \\ Universidad César Vallejo, Piura - Perú
}

\section{RESUMEN}

La presente investigación tuvo como propósito determinar la influencia de la estrategia aprendizaje basado en proyectos influye en el aprendizaje de las matemáticas en estudiantes de bachillerato, Guayaquil, Ecuador. 2021, con enfoque cuantitativo, tipo experimental, correlacional descriptivo, diseño cuasi experimental, utilizó el cuestionario aplicada 80 estudiantes. Determinando los resultados que la aplicación de la estrategia ABP., influyó en el desarrollo de los aprendizajes de las matemáticas, existiendo una diferencia significativa del 15,475 , entre los promedios de los grupos experimental y control, calculada con una t de Student de 17,386. Asimismo se determinó su influencia positiva en el desarrollo de la dimensión activación y regulación, apreciándose una diferencia significativa de 3,500, entre los promedios de los grupos experimental y control, con una t de Student calculada de 10,420; igualmente predomino en el desarrollo de la dimensión significatividad, evidenciando una diferencia reveladora de 5,725, entre 
los grupos experimental y control, con una t de Student calculada de 14,078; asimismo, influyó en la dimensión motivación por aprender, observándose una diferencia significativa entre los promedios de los grupos experimental y control de 5.650 con una $t$ de Student calculada de 14,078.

Palabras claves. estrategia; proyectos; aprendizaje; activación y regulación; significatividad. 


\title{
Project-based learning strategy influences the learning of mathematics in students, high school, Guayaquil, Ecuador. 2021
}

\begin{abstract}
The present research aimed to determine the influence of the project-based learning strategy influences the learning of mathematics in high school students, Guayaquil, Ecuador. 2021, with a quantitative approach, experimental type, descriptive correlational, quasi-experimental design, used the questionnaire applied by 80 students. Determining the results that the application of the ABP strategy, influenced the development of mathematics learning, with a significant difference of 15.475 , between the averages of the experimental and control groups, calculated with a Student's t of 17.386. Likewise, its positive influence on the development of the activation and regulation dimension was determined, showing a significant difference of 3,500, between the averages of the experimental and control groups, with a calculated Student $\mathrm{t}$ of 10,420; It also predominated in the development of the significance dimension, showing a revealing difference of 5.725 between the experimental and control groups, with a calculated Student $t$ of 14.078; Likewise, it influenced the motivation to learn dimension, observing a significant difference between the averages of the experimental and control groups of 5,650 with a calculated Student $t$ of 14,078 .
\end{abstract}

Keywords. strategy; projects; learning; activation and regulation; significance.

Artículo recibido: 30 noviembre. 2021 Aceptado para publicación: 29 diciembre 2021 Correspondencia: p7002291741@ucvvirtual.edu.pe Conflictos de Interés: Ninguna que declarar 


\section{INTRODUCCIÓN}

En la institución educativa, del contexto internacional, nacional y local, evidencian dificultades para aplicar conceptos de razonamiento lógico durante la vida práctica, afectando los aprendizajes, de acuerdo al nivel de complejidad. A pesar que existen estudios sobre los aprendizajes de las matemáticas, el problema está latente en los jóvenes. Los estudios realizados, indican que los aprendizajes de las matemáticas no son buenos, como se observa en los lugares que ocupan los países que intervienen en la prueba PISA, convirtiéndose en calidad de referente para evaluar el éxito de los sistemas educativos básicos. (OCDE, 2018). En Ecuador, el informe INEE, (2018), indica bajo rendimiento estudiantil en la capacidad de resolver problemas matemáticos, siendo un porcentaje del 70, $9 \%$, que no alcanzaron el nivel 2, frente al 23,4\% de alumnos de países miembros de la OCDE. Como las propuestas de estrategias quienes mejoraron las enseñanzas y los aprendizajes matemáticos y su comprensión, utilización en los ámbitos académicos, profesionales; fruto de ello, se conocen los trabajos de Polya, siendo de los primeros aportes al desarrollo de habilidades para resolver problemas de matemáticas. (Aguilar, 2014). Según Díaz-Barriga \& Hernandez (2005), se inicia como una corriente epistemológica cuyo interés estaba en esclarecer los problemas de la formación del conocimiento. Del mismo modo Kant, "Libro, critica de la razón pura, manifestando si el sujeto conoce la expresión de los objetos y fenómenos; es posible acceder al plano fenomenológico, mas no a la esencia de las “cosas en sî”. Según Tunnermann (2011), el punto de inicio de las concepciones constructivistas relacionadas con el conocimiento aplicados a la educación dadas por Piaget, manifiesta que el sujeto gradualmente va construyendo el conocimiento, por ende, crece en las habilidades intelectuales; asimismo, el contenido de la enseñanza y aprendizaje está provisto principalmente de conceptos, esquemas y estructuras de las ciencias, de esta manera se estimula el fortalecimiento de las capacidades de los alumnos. Días \& Brantley (2017) indica que el ABP, se encuentra en trabajos vistos por Dewey, sus principios básicos se pueden resumir en: un problema o pregunta desafiante, autenticidad, voz y elección de los estudiantes, critica y revisión. Por su parte McGrath (2002), destaca en su conceptualización que se trata de una estrategia que privilegia el trabajo grupal y la atención a problemas reales, estas condiciones inciden en que los estudiantes obtengan un aprendizaje cualitativamente 
menor al de aquellos que aprenden a través de métodos tradicionales. Así también Dewey (1993), citado por, Johari \& Hardsshaw, (2008), indica que aplicada estas actividades donde el sujeto tiene la oportunidad de reconstruir y reorganizar su experiencia en la medida que resignifica los elementos de su experiencia y va diseñando, siendo el alumno quien construye su aprendizaje. (pp.12). Alfaro et al., (2019) señalan el ABP, como una tarea compleja o problema de la realidad, para cuya resolución, el alumno debe desarrollar actividades de investigación, planificación, diseño, desarrollo, validación y testeo. Asimismo, el ABP, se define como un conjunto de actividades que obedecen a un propósito central, que es dar solución a un problema, obtener un producto tangible o no; para ello pone de manifiesto sus habilidades conocimientos y actitudes trabajando colaborativamente para lograr un producto final. (MINEDUC 2016). Sostiene Moncayo et al., (2020), activación y regulación, de diferentes tareas concebidas, se integran los procesos potenciando el carácter integral y su pertinencia para elevar la calidad del aprendizaje. Los autores definen el termino significatividad, como la formación de sentimientos, actitudes, valores, siendo primordial, en las resoluciones de problemas, a medida que se logra la significatividad del contenido, activándose en los sujetos la reflexión y regulación sobre el contenido. (Moncayo et al., 2020, pp. 270). Enfatizando en motivación por aprender, como el involucramiento continuo con actitud y a largo plazo asumiendo él mismo, en mantener esa actitud de por vida, se puede notar un estudiante que tienen tareas complejas, buscan solución con estructuras más lógicas y de análisis de alternativas. (Ames 1992, pp.7).

\section{ESTRATEGIAS METODOLÓGICAS O MATERIALES Y MÉTODOS}

La metodología de la investigación de tipo experimental, correlacional descriptivo; establece aquello que obtiene datos a través de la experimentación comparándolo con variables, determinando las causas y/o efectos de los fenómenos en estudio; concerniente a correlacional empareja variables de una población o grupo, estipulando de explicar el vínculo (Hernández 2010); fue de tipo correlacional descriptivo, describiéndose de forma aislada los resultados estableciendo la correlación entre cada variable. Contándose con diseño cuasi experimental, caracterizándose por la igualdad del propósito. No es posible la asignación aleatoria, de antemano, estos diseños, tienen semejanza a los experimentos, posibilitando estimar impactos del tratamiento, estímulo o programa, acotando de una apropiada comparación como base (p. 58) (Hernández 2014). Población estuvo 
constituida por 540, estudiantes del bachillerato Unidad Educativa, República de Venezuela. La muestra fueron 80 estudiantes de segundo de bachillerato ciencias A y B, divididos forma igual en grupos de; 40 estudiantes en el grupo control y 40 estudiantes en el grupo experimental; para el recoger la información la técnica utilizada fue la encuesta, técnica que es definida como un procedimiento que permite recoger opiniones, puntos de vista y percepciones en general de los investigados; el instrumento fue el cuestionario, el mismo que se construyó considerando las dimensiones de la competencia matemática que corresponde al aprendizaje que están cursando los alumnos; la validez de criterio, calculada con los datos de la prueba piloto; validez de constructo, obteniéndose con datos recogidos de prueba piloto, aplicada en la unidad educativa fiscal "Eduardo Flores Torres", con unidades de estudios similares, donde se determinó el grado de confiabilidad a través del Alfa de Crom Bach, cuya es de 0,775, y la magnitud es muy confiable. Aplicándose el pre prueba en ambos grupos, control y/o experimental, posteriormente al grupo experimental se aplicó el tratamiento, el mismo que consistió en la ejecución de quince actividades de aprendizaje; después se aplicó la post prueba, entre ambos grupos, demostrando el cambio esperado en la muestra seleccionada. Después los datos se trasladaron a tablas para el procesamiento respectivo; para la medición de datos se utilizaron tablas y figuras estadísticas, organizadas por variables y dimensiones, para el pre test y post test, en ambos grupos, tanto control y experimental, todo ello para el análisis descriptivo. Precisando el uso del software SPSS-versión 24, para determinar las tendencias utilizando estadística descriptiva, a fin de comprobar el aprendizaje en las matemáticas, de los participantes; También se determinó la comprobación de las hipótesis, dando lugar al análisis inferencial.

\section{RESULTADOS}

\section{Hipótesis general}

- La estrategia aprendizaje basado en proyectos influye significativamente en el aprendizaje de matemáticas de los estudiantes, bachillerato Guayaquil, Ecuador. 2021. 


\section{Tabla 1}

Prueba de hipótesis de la variable aprendizaje de las matemáticas.

\begin{tabular}{|c|c|c|c|c|c|c|c|c|c|}
\hline \multicolumn{10}{|c|}{ Diferencias emparejadas } \\
\hline & & \multirow[t]{2}{*}{ Media } & \multirow[t]{2}{*}{$\begin{array}{l}\text { Desviación } \\
\text { estandar }\end{array}$} & \multirow[t]{2}{*}{$\begin{array}{c}\text { Media de } \\
\text { error } \\
\text { estandar }\end{array}$} & \multicolumn{2}{|c|}{$\begin{array}{l}95 \% \text { de intervalo } \\
\text { de confianza de la } \\
\text { diferencia }\end{array}$} & \multirow[t]{2}{*}{$\mathbf{t}$} & \multirow[t]{2}{*}{ gl } & \multirow[t]{2}{*}{$\begin{array}{c}\text { Sig. } \\
\text { (bilate } \\
\text { ral) }\end{array}$} \\
\hline & & & & & Inferior & Superior & & & \\
\hline Par1 & $\begin{array}{l}\text { VD GE Postest } \\
\text { VD GC Postest }\end{array}$ & 15,475 & 5,630 & 0,890 & 13,675 & 17,275 & 17,386 & 39 & .000 \\
\hline
\end{tabular}

En las tablas 1, se aprecia que hay diferencia significativa entre los promedios de los grupos experimental y de control de 15,475 con t de Student calculada de 17,386 y una Sig. $=0,000$, por tanto, rechaza el Ho y se acepta la Hi; llegando a la conclusión que la estrategia aprendizaje basado en proyectos influyó significativamente en el aprendizaje de matemáticas en estudiantes de bachillerato, Guayaquil, Ecuador. 2021, comprobándose la efectividad del programa aplicado.

\section{Hipótesis especifica 1:}

- La estrategia aprendizaje basado en proyectos influye significativamente en la actividad y regulación de los estudiantes, Guayaquil, Ecuador. 2021.

\section{Tabla 2}

Prueba de hipótesis de la dimensión actividad y regulación

\begin{tabular}{|c|c|c|c|c|c|c|c|c|c|}
\hline \multicolumn{10}{|c|}{ Diferencias emparejadas } \\
\hline \multirow[b]{3}{*}{ Par 1} & & Media & $\begin{array}{c}\text { Desviación } \\
\text { estandar }\end{array}$ & $\begin{array}{l}\text { Media de } \\
\text { error } \\
\text { estandar }\end{array}$ & $\begin{array}{r}95 \% \text { de } \\
\text { de confi } \\
\text { dife }\end{array}$ & $\begin{array}{l}\text { intervalo } \\
\text { anza de la } \\
\text { encia }\end{array}$ & $\mathbf{t}$ & gl & $\begin{array}{l}\text { Sig. } \\
\text { (bilate } \\
\text { ral) }\end{array}$ \\
\hline & & & & & Inferior & Superior & & & \\
\hline & $\begin{array}{l}\text { VD GE } \\
\text { Postest } \\
\text { VD GC } \\
\text { Postest }\end{array}$ & 3,500 & 2,124 & ,336 & 2,821 & 4,179 & 10,420 & 39 & .000 \\
\hline
\end{tabular}

En las tablas 2, se aprecia que hay diferencia significativa entre los promedios de los grupos experimental y de control de 3,500 con t de Student calculada de 14,420 y una Sig. = 0,000, por tanto, de rechaza el Ho y se acepta la Hi; llegando a la conclusión que la estrategia aprendizaje basado en proyectos influyó significativamente en la actividad y regulación en estudiantes de bachillerato, Guayaquil, Ecuador. 2021, comprobándose la efectividad del programa aplicado. 


\section{Hipótesis especifica 2:}

- La estrategia aprendizaje basado en proyectos influye significativamente en la significatividad de los estudiantes, Guayaquil, Ecuador. 2021.

\section{Tabla 3}

Prueba de hipótesis de la dimensión significatividad

\begin{tabular}{|c|c|c|c|c|c|c|c|c|c|}
\hline \multicolumn{10}{|c|}{ Diferencias emparejadas } \\
\hline & & \multirow[t]{2}{*}{ Media } & \multirow[t]{2}{*}{$\begin{array}{l}\text { Desviación } \\
\text { estandar }\end{array}$} & \multirow[t]{2}{*}{$\begin{array}{l}\text { Media de } \\
\text { error } \\
\text { estandar }\end{array}$} & \multicolumn{2}{|c|}{$\begin{array}{l}95 \% \text { de intervalo de } \\
\text { confianza de la } \\
\text { diferencia }\end{array}$} & \multirow[t]{2}{*}{$\mathbf{t}$} & \multirow[t]{2}{*}{ gl } & \multirow[t]{2}{*}{$\begin{array}{l}\text { Sig. } \\
\text { (bilate } \\
\text { ral) }\end{array}$} \\
\hline & & & & & Inferior & Superior & & & \\
\hline Par 1 & $\begin{array}{l}\text { VD GE } \\
\text { Postest } \\
\text { VD GC } \\
\text { Postest }\end{array}$ & 5,725 & 2,572 & ,407 & 4,902 & 6,548 & 14,078 & 39 & .000 \\
\hline
\end{tabular}

En la tabla 3, se aprecia que hay diferencia significativa entre los promedios de los grupos experimental y de control de 5,725 con $\mathrm{t}$ de Student calculada de 14,078 y una Sig. = 0,000, por tanto, se rechaza el Ho y se acepta la Hi; llegando a la conclusión que la estrategia aprendizaje basado en proyectos influyó significativamente en la significatividad de estudiantes de bachillerato, Guayaquil, Ecuador. 2021, comprobándose la efectividad del programa aplicado

\section{Hipótesis especifica 3:}

- La estrategia aprendizaje basado en proyectos influye significativamente en la motivación por aprender en los estudiantes, Guayaquil, Ecuador. 2021.

\section{Tabla 4}

Prueba de hipótesis de la dimensión motivación por aprender

\begin{tabular}{|c|c|c|c|c|c|c|c|c|c|}
\hline \multicolumn{10}{|c|}{ Diferencias emparejadas } \\
\hline & & \multirow[t]{2}{*}{ Media } & \multirow[t]{2}{*}{$\begin{array}{c}\text { Desviación } \\
\text { estandar }\end{array}$} & \multirow[t]{2}{*}{$\begin{array}{c}\text { Media de } \\
\text { error } \\
\text { estandar }\end{array}$} & \multicolumn{2}{|c|}{$\begin{array}{c}95 \% \text { de intervalo } \\
\text { de confianza de la } \\
\text { diferencia }\end{array}$} & \multirow[t]{2}{*}{$\mathbf{t}$} & \multirow[t]{2}{*}{ gl } & \multirow[t]{2}{*}{$\begin{array}{l}\text { Sig. } \\
\text { (bilate } \\
\text { ral) }\end{array}$} \\
\hline & & & & & Inferior & Superior & & & \\
\hline Par 1 & $\begin{array}{l}\text { VD GE } \\
\text { Postest } \\
\text { VD GC } \\
\text { Postest }\end{array}$ & 5,650 & 2,315 & ,436 & 4,909 & 6,391 & 14,078 & 39 & .000 \\
\hline
\end{tabular}

En la tabla 4, se aprecia que hay diferencia significativa entre los promedios de los grupos experimental y de control de 5,650 con t de Student calculada de 14,078 y una Sig. = 0,000, por tanto, de rechaza el Ho y se acepta la Hi; llegando a la conclusión que la 
estrategia aprendizaje basado en proyectos influyó significativamente la motivación por aprender en estudiantes de bachillerato, Guayaquil, Ecuador. 2021, comprobándose la efectividad del programa aplicado

\section{RESULTADOS Y DISCUSIÓN}

La estrategia del ABP., tiene efectos positivos en el aprendizaje de matemáticas de los estudiantes de bachillerato, Guayaquil, Ecuador. 2021. Para el objetivo general: Determinar la influencia de la estrategia aprendizaje basado en proyectos en el aprendizaje de matemáticas, en la tabla 02, se evidencia que el grupo control se ubicó en nivel medio (50\%) en el pre test y en el postest se mantiene en el mismo; a diferencia del grupo experimental se ubicó en el nivel medio del pre test (57,5\%) y se ubicó con el $100 \%$ en nivel alto de post test.

De la misma manera, en las tablas 8 y 9 , en los resultados inferenciales, existe diferencia de medias del 15,475, entre los grupos control y experimental, calculada con t de Student de 17,386, Sig. =0,000. El hallazgo se fundamenta, Tuston, (2020), en su trabajo de investigación donde concluye que la estrategia $\mathrm{ABP}$ fortalece la disponibilidad del estudiante hacia los aprendizajes de las matemáticas. Se corrobora con la teoría de (Vygotski, 1988), sociocultural, indica que cada persona mediante la interacción con el otro, construye su propio conocimiento, buscando oportunidades para emitir lo que sabe, probar y negociar dentro del contexto. También sustenta, Piaget (1997), que el sujeto gradualmente va construyendo el conocimiento. Enfatizando, Ausubel (1993), que el aprendizaje como la habilidad para relacionarse con los nuevos saberes con los existentes en la estructura cognitiva; complementando con materiales y la motivación, teniendo en cuenta los criterios de individualidad relacionados a los ritmos y estilos de aprendizaje. La investigación de Flores \& Juárez, (2017), quienes cuenta que el 63\% de educandos se encontraban en el nivel pre formal; a diferencia que al culminar el proceso el $90 \%$. se ubicó en nivel estratégico esperado, donde los sujetos cuentan con predisposición para el trabajo en equipo. En la indagación realizada por Barreto (2018), los resultados obtenidos, guarda similitud con la investigación, confirmando, que el ABP., de las matemáticas mejoro el rendimiento académico, favoreciendo al estudiantado, porque dieron un extraordinario cambio en la adquisición de sus aprendizajes, logrando las competencias propuestas. 
En cuanto al objetivo específico 01: Determinar la influencia del ABP., en la activación y regulación. La tabla 3, se observa que el grupo control se ubica con 55\%, nivel alto del pretest, y se mantiene con el mismo nivel y porcentaje en el postest. En cambio, el grupo de experimentación, a inicio obtuvo como propuesta el nivel medio (63\%) del pretest; luego se ubica nivel alto del postest (100\%). Igualmente, las tablas $10 \mathrm{y} 11$, evidencian que existe diferencia de medias de 3,500 entre grupos, calculada con t de Student de 10,420 y una Sig. $=$ 0,000. Los hallazgos se corroborarán, Moncayo et al., (2020) sostienen que la activación y regulación y su pertinencia para elevar la calidad del aprendizaje, tiene como elementos que están presentes en la resolución de problemas, el pensamiento y la memoria. El estudio coincide con, Díaz (2016) que los estudiantes mejoraron en su motivación, participación y aprendizajes, convirtiéndose en protagonista activos de sus conocimientos. Con los aportes de Rivas, (2007) procesos cognitivos son habilidades mentales que el ser humano, desarrolla al realizar cualquier actividad, mediante la percepción, atención y memoria.

Lo relacionado al objetivo específico 02: Determinar la influencia de la estrategia basada en proyectos, dimensión significatividad. La tabla 4, evidencia $62 \%$ del grupo control se ubica en un alto nivel del pretest; y se mantiene con el mismo porcentaje y nivel al postest. A diferencia, al grupo experimental ubicándose en nivel medio relacionado con el pretest $(50 \%)$ y después se ubica en nivel alto con el 100\%. En el análisis inferencia las tablas 12 y 13, demuestran la existencia de diferencia relevante de 5,725 entre los promedios de grupos experimental y de control, con 14,078 calculada por t de Student y una Sig. = 0,000, concluyendo que la congruencia en las estrategias los ABP., influye para desarrollar la significatividad, Serrano \& Pons (2011). El significado que van dando los sujetos a los hechos, deben ir de la mano con los significados, que orienta a generar las condiciones propicias articulando los nuevos saberes y lo que ya está en su mente. MINEDUC (2016) La competencia de resolución de problemas centrada en los aprendizajes, a través de reglas que permitan aplicar ecuaciones, como también dar soluciones en situaciones polémicas. Afirma Skatkin (1985), a medida que se logra la significatividad, se promueve en el estudiante la reflexión, regulación de la actividad, así como el control valorativo de sus propias acciones, relacionados con el contexto real de formación, que permite corregir, ajustar sus errores y regular su actividad. Podemos evidenciar con el estudio investigativo de Herrera (2017), quien da su testimonio, se pudo 
mostrar la significatividad entre el pre test y el pos test, demostrando pertinencia entre la formación didáctica y el ABP.

Asimismo, para el objetivo específico 03: Determinar la influencia de la estrategia ABP., en la dimensión, motivación por aprender. En la tabla 5, como podemos visualizar que $53 \%$ grupo control está ubicado en nivel medio, más $42 \%$ en nivel alto, del pretest, y luego el 52\% se ubica en nivel medio del postest. En tanto, el 58\% perteneciente al grupo experimental encentrándose en nivel medio del pretest; luego se ubica con el $100 \%$ en nivel alto para el postest. De la misma manera, las tablas 14 y 15, presentan la evidencia de la existencia de diferencia de 5,650, resaltando los promedios de los grupos experimental y de control con t de Student calculada de 14,078 y una Sig. $=0,000, \mathrm{El}$ presente trabajo reafirma según Ames (1992), motivación por aprender como el involucramiento continuo, con actitud y a largo plazo, asumiendo él, en mantener esa actitud de por vida. También es similar con el estudio de Ajello (2003). Donde dice que el despertar el interés para continuar aprendiendo va a depender del aliciente, donde actué con autonomía. La semejanza investigación realizada con Izaguirre et al. (2020) concluyen que la estrategia que aplico fue motivadora, debido que los alumnos crecieron efectivamente, visualizando la aplicación de ideas, igual afianzan los principios matemáticos y puntos de vista, para sustentar las soluciones que habían propuesto, investigación similar al estudio realizado, donde los aprendices, en su totalidad están motivados para aprender, demostrando mejoras en sus aprendizajes.

\section{CONCLUSIÓN O CONSIDERACIONES FINALES}

Se determinó que las estrategias aprendizaje basadas en proyectos, confirmándose significativamente el progreso del aprendizaje de matemática, de los estudiantes, bachillerato, Guayaquil, Ecuador. 2021, determinando una afinidad significativa del 15,475 con una $\mathrm{T}=17,386$. y un Sig.=0.000, permitiéndose aceptabilidad de la $\mathrm{H}_{\mathrm{i}} \mathrm{y}$ se rechaza la Ho, comprobando la eficacia de las estrategias, vital importancia para la expansión de los aprendizajes matemáticos, en relación al apoyo del estudiante, contribuyendo a actuar con actitud en la resolución de problemas por propia iniciativa.

Se determinó que las estrategias ABP, desarrolla la activación y regulación de los estudiantes de bachillerato, 3,500 determinando una semejanza relevante del 10.420, calculada con $\mathrm{T}$ de Student, (tabla 11), por lo visto se admite la $\mathrm{H}_{\mathbf{i}}$ desestimando $\mathrm{H}_{\mathbf{0}}$, constatando la equivalencia del ABP, en la actualidad la sociedad requiere de pensantes 
capaces de transformar el conocimiento adquirido en nuevos aprendizajes, mas no requiere únicamente repetidores de información.

Se determinó que las estrategias ABP., desarrolla la significatividad, estableciendo una disconformidad significativa del 5,725 en concordancia 14,078 calculado con la $\mathrm{T}$ de Student, (tabla 13), comprobándose la ascendencia del ABP, hallándose los alumnos en un alto nivel, por tanto, se admite la $\mathrm{H}_{\mathrm{i}} \mathrm{y}$ se declina la $\mathrm{H}_{0}$, verificándose la operatividad relacionada a significatividad de los aprendizajes, el sujeto adquiere, familiariza los aprendizajes nuevos, relacionándolos con su experiencia, buscando de manera natural, la relación de las ideas abstractas existentes en un mundo real.

Se demuestra que el ABP., desarrolla la motivación por aprender, estableciendo una diferencia 5.650 significativa, calculada de 14,078 con T de Student, (tabla 15), contando con el rendimiento del grupo de control revelan el postest a un nivel medio del 52\%, en cambio se viene situando en un nivel alto al grupo experimental, con el $100 \%$, comprobándose que el ABP, trabajado, acepta la $\mathrm{H}_{\mathbf{i}}$ favorable en los estudiantes. Rechazándose la $\mathrm{H}_{0}$, permitiendo que las estrategias fueron eficaces, por la estimulación recibida, facilitando que los escolares permanezcan comprometidos, motivados durante la realización de actividades, fortaleciendo sus aprendizajes con mucha autonomía.

\section{LISTA DE REFERENCIAS}

Alfaro Cardenas, J. L. (2021). Estrategia de enseñanza y el aprendizaje de la matematica en el estudiantes de una universidad publica. Lima, Perú Universidad Cesar Vallejo.

https://hdl.handle.net/20.500.12692/69280

Anaya Durand, A., Anaya,Huertas, C. (2010). ¿Motivar para aprobar o para aprender? Estrategias de motivación del aprendizaje para los estudiantes: Tecnología, Ciencia, Educación, vol. 25. 5-14. Disponible en:

http://www.redalyc.org/articulo.oa?id=48215094002

Arnal, Justo., Rincón, Delio., La Torre, Antonio. (1992) Investigación educativa: Fundamentos y metodología, E Sant Adrià de Besos, Barcelona, editorial Labor, https://www.icmujeres.gob.mx/wpcontent/uploads/2020/05/InvestigacionEducativa.[pdf].

Barreto Manihuari, E. F. (2018). "El aprendizaje basado en problemas de las matemáticas en la mejora del rendimiento académico en estudiantes del 1er ciclo en la 
Universidad Tecnológica del Perú, 2017-II.” Universidad César Vallejo. https://repositorio.ucv.edu.pe/bitstream/handle/20.500.12692/13676/Barreto_ME F.pdf?

Campanario Juan , M., Otero, Isabel., Brincones Calvo, I. (2000) «Más allá de las ideas previas como dificultades de aprendizaje: las pautas de pensamiento, las concepciones epistemológicas y las estrategias meta cognitivas de los alumnos de ciencias». Enseñanza de las ciencias: revista de investigación y experiencias didácticas, [en línea], 2000, Vol. 18, n. ${ }^{\circ} 2$.

https://raco.cat/index.php/Ensenanza/article/view/52234 [Consulta: 25-11-2021].

Castañeda, A., González, J., y Mendo, L. (2017). Libros de Matemáticas para primer grado de secundaria en México: problemas y estrategias de solución. Revista Electrónica de Investigación Educativa, 19(4),97-111. https://doi.org/10.24320/redie.2017.19.4.1173https://www.terras.edu.ar/bibliotec a/11/11DID_Chevallard_Unidad_3.pdf

Cobo y Valdivia. (2017). Aprendizaje Basado en Proyectos. Pontificia Universidad Católica del Perú. Instituto de Docencia Universitaria. https://idu.pucp.edu.pe/wpcontent/uploads/2017/07/5.-aprendizaje.pdf.

Currículo, (2016). Currículo de educación general Básica. Ministerio de Educación. Ecuador. https://educacion.gob.ec/curriculo-educacion-general-basica/

Díaz-Barriga, F., Hernández, G. (2005), Estrategias docentes para un aprendizaje significativo. Una interpretación constructivista. 2da Edición. Mc Graw Hill. https://buo.org.mx/assets/diaz-barriga\%2

García Peñalvo, F. (2017). Aprendizaje basado en proyectos (ABP): evaluación desde la perspectiva de alumnos de Educación Primaria. Revista de Investigación Educativa, 35(1), 113-131. DOI:http://dx.doi.org/10.6018/rie.35.1.2468

García Gonzales M., Varela de Montoya, H., Sifontes Valdés, B., y Peña Rubio, C. (2014). Significación del enfoque histórico-cultural de Vygotsky para el tratamiento de las relaciones interdisciplinarias. Hum Med vol.14 no.2, pp. 1-14

http://scielo.sld.cu/pdf/hmc/v14n2/hmc14214.[pdf]

Gomez Chocon, I. (2005). Reseña de "Matemática emocional. Los afectos en el aprendizaje matemático" de Inés María Gómez Chacón. In Educación Matemática (Vol. 17, Issue 1, 185-189).https://books.google.es/books?id=hik- 
$\underline{\text { KLZ9SYkC\&printsec }=\text { frontcover } \& \mathrm{hl}=\mathrm{es} \# \mathrm{v}=\text { onepage } \& \mathrm{q} \& \mathrm{f}=\text { false }}$

Gómez, Samaniego, G. M. (2020). Modelo de estrategia didáctica para fortalecer el aprendizaje de matemática en estudiantes de segundo bachillerato, Educativa Vicente Rocafuerte, Ecuador-2 [Tesis doctoral, Universidad Cesar Vallejo, Perú].

https://repositorio.ucv.edu.pe/bitstream/handle/20.500.12692/69281/G\%C3\%B3 $\underline{\text { mez_SGM-SD.pdf?sequence }=1 \& \text { isAllowed }=\mathrm{y}}$

Godino, J. D., Aké, L. P., Contreras, A., Díaz, C., Estepa, A., F. Blanco, T., Lacasta, E., Lasa, A., Neto, T., Oliveras, M. L. y Wilhelmi, M. R. (2015). Enseñar en primera línea: uso de Internet y aprendizaje basado en problemas para mejorar la enseñanza en el aula, enseñanza de las Ciencias, 33.1, pp. 127-150. http://dx.doi.org/10.5565/rev/ensciencias.1468

Instituto Nacional de Evaluación Educativa. (2018). Educacion en Ecuador. Resultados de PISA para el Desarrollo.[Education in Ecuador. Results of PISA for Development]. OECD Reports, 152. https://n9.cl/ofgk3.

Johari, Ababas. \& Bradshaw, Amy, C. (2008 Aprendizaje basado en proyectos en un programa de pasantías: un estudio cualitativo de roles relacionados y sus atributos motivacionales. Investigación y desarrollo de tecnología educativa. 56, 329-359. DOI 10.1007/s11423-0069009-228.Kaur, B. (2010). Hacia la excelencia en la educación matemática: la experiencia de Singapur. Procedia Ciencias sociales y del comportamiento.

file:///C:/Users/Jácome/Downloads/Towards_Excellence_in_Mathematics_Educati onSingap.pdf.

Leal Uisi, S. y Bong Anderson, S.(2015) La resolución de problemas matemáticos en el contexto de los proyectos de aprendizaje. Revista de Investigación. 2015;39(84):71-93.[fecha de Consulta 28 de Mayo de 2021].ISSN:0798-0329.

Disponible en: https://www.redalyc.org/articulo.oa?id=376140399004.

Ministerio de educación (2016) Currículo 2016. https://educacion.gob.ec/curriculo/ Miranda CH., Wilhelm K., Martin G., Arancibia M y Osses S. (2013) Autoestima professional em professores beneficiários do Programa de Pós-título de Matemática no contexto da avaliação docente. Universidad de Chile vol.39 no.1.129-142. 
http://dx.doi.org/10.4067/S0718-07052013000100008

Ministerio de Educación del Ecuador [MinEduc]. (2019). Currículo de los niveles de Educación Obligatoria. Nivel Bachillerato-tomo 1.481. https://educacion.gob.ec/wp-content/uploads/downloads/2019/09/BGU-tomopdf?fbclid=IwAR0UfOT0sT4HqDdXcNGna1tNbFc1j_XS3oQ46Bz7GdrHVuL 4n_SGtCgr910

Ministerio de Educación del Ecuador [MinEduc]. (2019). Currículo de los niveles de Educación Obligatoria. Nivel bachillerato-tomo 2.482. https://educacion.gob.ec/wp-content/uploads/downloads/2019/09/BGU-tomo2.pdf?fbclid=iwar0ufot0st4hqddxcngna1tnbfc1j_xs3oq46bz7gdrhvul4n_sgtcgr9 10

Moncayo Carreño, O. F., Torres Moreno, V. E., Martínez Roselló, M. M., Pupo Cejas, Y., Tiá Pacheco, M. L., \& Pérez,G. (2020). La activación regulación del aprendizaje en la asignatura Fundamentos de la Administración. Revista Conrado, 16(74), 266-275. $\quad$ http://scielo.sld.cu/pdf/rc/v16n74/1990-8644-rc-16-74266. [pdf]

OCDE (2018) Programa para la Evaluación Internacional de Alumnos- PISA- Resultado. https://www.oecd.org/pisa/pisa-fordevelopment/PISA_D_Resultados_en_Foco.pdf

Osses Bustingorry, S. \& Jaramillo S.(2008) Metacognicion: Un Camino para aprender a aprender, Estudios pedagógicos (Valdivia), v.34 n.1, 187-197.

http://dx.doi.org/10.4067/S0718-07052008000100011

Ortiz Granja, D. (2015). El constructivismo como teoría y método de enseñanza. Sophia, Colección de Filosofía de la Educación, (19),93-110.[fecha de Consulta 30 de Mayo de 2021]. ISSN: 1390-3861. Disponible en: https://www.redalyc.org/articulo.oa?id=441846096005

Prieto, Gerardo y Delgado, Ana. (2010). Fiabilidad y Validez. Revista papeles del psicólogo, 2010. Vol. 31(1), pp. http://www.papelesdelpsicologo.es/pdf/1797.[pdf]

Pujol Cunill, F. (2017). El Aprendizaje Basado en Proyectos y el aprendizaje por descubrimiento guiado como estrategias didácticas en Biología. Disponible en: https://reunir.unir.net/handle/123456789/6052 
Quishpe Pilc, M. (2021), investigó: "La enseñanza de las matemáticas basado en la resolución de problemas como fundamento del aprendizaje significativo de las funciones exponenciales.

http://repositorio.puce.edu.ec/bitstream/handle/22000/18692/Quishpe\%20PilcoTesis.pdf? sequence $=1 \&$ is Allowed $=\mathrm{y}$

Rivas, Axel., y Scasso, Martin. (2017). ¿Qué países mejoraron la calidad educativa? América Latina en las evaluaciones de aprendizajes. CPPEC. Políticas Públicas. https://www.cippec.org/wp-content/uploads/2017/12/DT-Que-paisesmejoraronen-PISA-vf.[pdf]

Rosales Brenda A., Flores Medrano E. y Escudero Avila, D. (2018). Aprendizaje Basado en Proyectos: Explorando la caracterización personal del profesor de matemáticas. Zetetiké, Campinas, v.26, n.3, p.506-525. DOI: https://doi.org/10.20396/zet.v26i3.8650908 